\title{
Moduli Space of Topological 2-form Gravity
}

\author{
Mitsuko Abe*, Akika Nakamichi ${ }^{\dagger}$ and Tatsuya Ueno ${ }^{\ddagger}$ \\ Department of Physics, Tokyo Institute of Technology \\ Oh-okayama, Meguro-ku, Tokyo 152, Japan \\ TIT/HEP-224/COSMO-32 June, 1993
}

\begin{abstract}
We propose a topological version of four-dimensional (Euclidean) Einstein gravity, in which anti-self-dual 2-forms and an SU(2) connection are used as fundamental fields. The theory describes the moduli space of conformally self-dual Einstein manifolds. In the presence of a cosmological constant, we evaluate the index of the elliptic complex associated with the moduli space.
\end{abstract}

\footnotetext{
*e-mail: mabe@phys.titech.ac.jp

$\dagger$ e-mail: akika@phys.titech.ac.jp

‡e-mail: tatsuya@phys.titech.ac.jp
} 
Topological gravity is a field theoretic description of the moduli space of gravitational instantons. Such a theory was first considered by Witten [1], where a topological version of conformal gravity in four dimensions was presented as a gravitational analogue of topological Yang-Mills theory (TYMT) [2]. The moduli space of the theory, the space of conformally self-dual gravitational instantons, was investigated in detail by Perry and Teo [3]. Since the work of Witten there have been several attempts to construct such four-dimensional topological theories modeling different gravitational moduli spaces [4]-[8]. In Ref. [9], we proposed a topological version of four-dimensional (Euclidean) Einstein gravity with or without a cosmological constant. This topological version is obtained by modifying an alternative formulation of Einstein gravity developed by Capovilla et al. [10], in which anti-self-dual 2-forms and an $\mathrm{SU}(2)$ connection are used as fundamental fields, instead of the metric or the tetrad. With an appropriate choice of gauge condition, the BRST invariant quantum action of the topological theory becomes the classical Einstein action plus ghost terms which cancel out all local degrees of freedom. However there still remain zero-modes in the quantum action. The (finite) number of them is closely related to the dimension of the moduli space, which now consists of Einstein manifolds with self-dual Weyl tensor. When the cosmological constant is non-zero, the moduli space is up to orientation, identical with the one considered by Torre in which the Weyl tensor is anti-self-dual [4. In his paper the dimension of the moduli space is found to be zero when the cosmological constant is positive, and the result is true also in our case. In the case of non-zero cosmological constant, we evaluate the index of an elliptic complex associated with our moduli space by applying the Atiyah-Singer index theorem. We also discuss the case of vanishing cosmological constant and mention the dimension of the moduli space on the $K 3$ surface.

We start with fundamental fields, a trio of 2-forms $\Sigma^{k}$, which transform under the chiral local-Lorentz representation $(2,0)$ of $\mathrm{SU}(2)_{L} \times \mathrm{SU}(2)_{R}$, and a connection 1-form $\omega^{k}$ associated with the $\mathrm{SU}(2)_{L}$. It is shown that $\Sigma^{k}$ and $\omega^{k}$ are anti-self-dual 
with respect to the $\mathrm{SO}(4)$ indices, when expressed with them instead of the $\mathrm{SU}(2)$ indices $i, j, k, \cdots$, 11. We take $\Sigma^{k}$ as primary metric fields to define the metric $g_{\mu \nu}$ on a four-dimensional oriented manifold $M_{4}$, 円

$$
g^{\frac{1}{2}} g_{\mu \nu}=-\frac{1}{12} \epsilon^{\alpha \beta \gamma \delta} \Sigma_{\mu \alpha} \cdot\left(\Sigma_{\beta \gamma} \times \Sigma_{\delta \nu}\right), \quad g \equiv \operatorname{det}\left(g_{\mu \nu}\right)
$$

We propose the following action for topological gravity with cosmological constant $\Lambda[9]$,

$$
S_{T G}=\int_{M_{4}} \Sigma^{k} \wedge F_{k}-\frac{\Lambda}{24} \Sigma^{k} \wedge \Sigma_{k}
$$

where $F^{k} \equiv d \omega^{k}+(\omega \times \omega)^{k}$. Varying the action with respect to each of fields $\Sigma^{k}$, $\omega^{k}$, we obtain the equations of motion:

$$
\begin{gathered}
F^{k}-\frac{\Lambda}{12} \Sigma^{k}=0, \\
D \Sigma^{k}=d \Sigma^{k}+2(\omega \times \Sigma)^{k}=0 .
\end{gathered}
$$

For the $\Lambda \neq 0$ case, eliminating $\Sigma^{k}$ from the action by using (3a) we obtain the effective action proportional to $\int F^{k} \wedge F_{k}$, which is the classical action of the TYMT for the $\mathrm{SU}(2)$ gauge group [12]. On the other hand, for the $\Lambda=0$ case, the action describes a BF-type topological field theory [13], 114. In this topological model, there exists a symmetry generated by a parameter 1 -form $\theta^{k}$ in addition to the local-Lorentz (with a 0-form $\phi^{k}$ ) and diffeomorphism (with a vector field $\xi^{\mu}$ ) symmetries,

$$
\delta \omega^{k}=D \phi^{k}+\tilde{\mathcal{L}}_{\xi} \omega^{k}+\frac{\Lambda}{12} \theta^{k}, \quad \delta \Sigma^{k}=2(\Sigma \times \phi)^{k}+\tilde{\mathcal{L}}_{\xi} \Sigma^{k}+D \theta^{k},
$$

where we have used a modified action $\tilde{\mathcal{L}}_{\xi}$ for diffeomorphism, which differs from usual Lie derivative $\mathcal{L}_{\xi}$ by the local-Lorentz transformation $\delta_{\tilde{\phi}}$ with a parameter $\tilde{\phi}^{k}=\xi^{\mu} \omega_{\mu}^{k}: \quad \tilde{\mathcal{L}}_{\xi}=\mathcal{L}_{\xi}-\delta_{\tilde{\phi}}$ [6]. The $\theta^{k}$-symmetry is regarded as a 'restricted' topological symmetry which preserves the equations of motion (3). With the appearance of the $\theta^{k}$-symmetry, the theory turns out to be on-shell reducible in the

\footnotetext{
${ }^{1}$ Greek indices $\mu, \nu, \cdots$, denote world indices on $M_{4}$ while Latin indices $a, b, \cdots$, the $\mathrm{SO}(4)$ indices. We use the notation for the $\mathrm{SU}(2)$ indices, $F \cdot G \equiv F^{i} G^{i}$ and $(F \times G)^{i} \equiv \varepsilon_{i j k} F^{j} G^{k}$, where $\varepsilon_{i j k}$ is the structure constant of $\mathrm{SU}(2)$.
} 
sense that the transformation laws (伍) are invariant under

$$
\delta \phi^{k}=-\frac{\Lambda}{12} \epsilon^{k}, \quad \delta \theta_{\mu}^{k}=D_{\mu} \epsilon^{k}+2 \rho^{\nu} \Sigma_{\nu \mu}^{k}, \quad \delta \xi^{\mu}=-\rho^{\mu},
$$

as long as the equations of motion are satisfied. The transformations with parameters $\epsilon^{k}$ and $\rho^{\mu}$ correspond to redundant local-Lorentz and diffeomorphism symmetries respectively.

Our strategy to construct a topological quantum field theory is to consider the following equation as a gauge fixing condition for the $\theta^{k}$-symmetry (except for the redundant part of the symmetry):

$$
{ }^{t f} \cdot \Sigma^{i} \wedge \Sigma^{j} \equiv \Sigma^{(i} \wedge \Sigma^{j)}-\frac{1}{3} \delta_{i j} \Sigma^{k} \wedge \Sigma_{k}=0
$$

This quadratic equation for $\Sigma^{k}$ is the constraint imposed in original 2-form Einstein gravity [10] and is a necessary and sufficient condition that $\Sigma^{k}$ is composed of tetrad 1-form: $\Sigma^{k}(e)=-\eta_{a b}^{k} e^{a} \wedge e^{b}$, where $\eta_{a b}^{k}$ are anti-self-dual constant coefficients called the t'Hooft's $\eta$ symbols [15]. Such a 2-form $\Sigma^{k}(e)$ is anti-self-dual with respect to world indices and via (3a), the curvature $F^{k}$ also becomes anti-self-dual. The set of equations in (3),(6) arose before as an ansatz within the framework of 2-form Einstein gravity with the cosmological constant [10], [16]. We consider them to be instanton equations. Here we assume a tensor-SU(2) connection $\nabla_{\mu}$ compatible with $\Sigma^{k}$, that is, $\nabla_{\mu} \Sigma_{\nu \rho}^{k}=0$, which leads to the metricity condition. Then (3b) and (6) make the anti-self-dual part of torsion vanish. We take a torsion-free extension to the self-dual part in order to work on a Riemannian manifold. In the case we obtain the following relations between the $s u(2)$ valued curvature $F^{k}$ and the anti-self-dual parts of Riemann curvature tensor and Weyl tensor as follows:

$$
{ }^{(-)} R_{\mu \nu \rho \tau}=4 F_{\mu \nu} \cdot \Sigma_{\rho \tau}, \quad \quad{ }^{(-)} W_{\mu \nu \rho \tau}=4\left(F_{\mu \nu}-\frac{\Lambda}{12} \Sigma_{\mu \nu}\right) \cdot \Sigma_{\rho \tau}
$$

From (3a) and (7), the Einstein equation $R_{\mu \nu}=\Lambda g_{\mu \nu}$ and ${ }^{(-)} W_{\mu \nu \rho \tau}=0$ are derived. So our instanton equations determine conformally self-dual Einstein manifolds. Note that in the case $\Lambda=0$, the Ricci tensor becomes zero as well as ${ }^{(-)} W_{\mu \nu \rho \tau}$, or equivalently the Riemann tensor is self-dual, ${ }^{(-)} R_{\mu \nu \rho \tau}=0$. 
Since our topological model has on-shell reducible symmetries (5), it is slightly complicated to quantize it by the BRST gauge-fixing procedure, and so we adopt the Batalin-Fradkin-Vilkovisky (BFV) formalism [17], 18]. In this canonical formalism we first obtain the nilpotent BRST charge in the extended phase space. Using the BRST charge and gauge conditions for various symmetries in the model, the partition function is defined as a path integral with the BRST invariant quantum action $S_{q} \equiv \int d t d^{3} x\left[\dot{\varphi} \cdot \mathcal{P}_{\varphi}-H_{e f f}\left(\varphi, \mathcal{P}_{\varphi}\right)\right]$, where $\varphi$ and $\mathcal{P}_{\varphi}$ are fields and their canonical momenta, respectively and $H_{e f f}$ is the BRST invariant effective Hamiltonian. Finally, integrating out conjugate momenta, we obtain the completely covariant form of the quantum action $S_{q}$. It consists of the classical action $S_{T G}$ plus gauge fixing and ghost terms, $\int \psi_{i j} \Sigma^{i} \wedge \Sigma^{j}+\cdots$, where the term with a symmetric trace-free Lagrange multiplier field $\psi_{i j}$ comes from the gauge fixing term in (6). The sum $S_{T G}+\int \psi_{i j} \Sigma^{i} \wedge \Sigma^{j}$ is just the original action of 2-form Einstein gravity [10], and hence the action $S_{q}$ becomes the classical Einstein action plus other terms which cancel out local degrees of freedom. In $S_{q}$ there arise cubic (and higher than cubic) ghost terms, which never emerge from the usual Faddeev-Popov procedure. This BRST quantization is reported in detail in 19.

To investigate the number of zero-modes in the quantum action $S_{q}$, we consider the moduli space $\mathcal{M}$ defined by our instanton equations (3),(6) describing conformally self-dual Einstein manifolds. Given a solution $\left(\Sigma_{0}^{k}, \omega_{0}^{k}\right)$ of the instanton equations, the tangent space $T(\mathcal{M})$ of $\mathcal{M}$ at the point is identical with the space of infinitesimal fluctuations $\left(\delta \Sigma^{k}, \delta \omega^{k}\right)$ which satisfy linearized instanton equations,

$$
D_{1}\left(\delta \Sigma^{k}, \delta \omega^{k}\right) \equiv\left(D \delta \omega^{k}-\frac{\Lambda}{12} \delta \Sigma^{k} ; D \delta \Sigma^{k}+2\left(\delta \omega \times \Sigma_{0}\right)^{k} ; 2^{t f \cdot} \Sigma_{0}^{i} \wedge \delta \Sigma^{j}\right)=0,
$$

modulo fluctuations generated by the local-Lorentz $(\mathrm{SU}(2))$ transformation and diffeomorphism:

$$
T(\mathcal{M})_{\left(\Sigma_{0}^{k}, \omega_{0}^{k}\right)}=\left\{\left(\delta \Sigma^{k}, \delta \omega^{k}\right) \mid D_{1}\left(\delta \Sigma^{k}, \delta \omega^{k}\right)=0\right\} /\{S U(2) \times \text { diffeo. }\}
$$

We define the following sequence of mappings on a compact conformally self-dual 
Einstein manifold:

$$
0 \stackrel{D_{-1}}{\rightarrow}\left\{\begin{array}{c}
\text { space of } \\
\left(\phi^{k}, \xi^{\mu}\right)
\end{array}\right\} \stackrel{D_{\rho}}{\rightarrow}\left\{\begin{array}{c}
\Omega_{1} \\
\text { space of } \\
\left(\delta \Sigma^{k}, \delta \omega^{k}\right)
\end{array}\right\} \stackrel{D_{1}}{\rightarrow}\left\{\begin{array}{c}
\text { space of } \\
\text { instanton equations }
\end{array}\right\} \stackrel{D_{2}}{\rightarrow} 0 .
$$

In the above sequence $D_{-1}$ and $D_{2}$ are identically zero operators. The operator $D_{0}$ is defined by

$$
D_{0}\left(\phi^{k}, \xi^{\mu}\right) \equiv\left(2\left(\Sigma_{0} \times \phi\right)^{k}+\tilde{\mathcal{L}}_{\xi} \Sigma_{0}^{k} ; D \phi^{k}+\tilde{\mathcal{L}}_{\xi} \omega_{0}^{k}\right)
$$

We can easily check that $D_{1} D_{0}=0$ and the ellipticity of the sequence (10). Hence it is an elliptic complex. Defining the inner product in each space $\Omega_{i}$, we can introduce the adjoint operators $D_{0}^{*}$ and $D_{1}^{*}$ for $D_{0}$ and $D_{1}$ respectively and the Laplacians $\triangle_{i} ; \triangle_{0}=D_{0}^{*} D_{0}, \triangle_{1}=D_{0} D_{0}^{*}+D_{1}^{*} D_{1}, \triangle_{2}=D_{1} D_{1}^{*}$. We may then define the cohomology group on each $\Omega_{i}$,

$$
H^{i} \equiv \operatorname{Ker} D_{i} / \operatorname{Im} D_{i-1}
$$

It is easy to show that $H^{i}$ is equivalent to the kernel of $\triangle_{i}$, the harmonic subspace of $\Omega_{i}$. These cohomology groups are finite-dimensional and we set $h^{i} \equiv \operatorname{dim} . H^{i}$. The $H^{1}$ is exactly identical with the tangent space of $\mathcal{M}$ in (9), the dimension of which we need to know. On the space $\Omega_{0}, H^{0}$ is equal to Ker $D_{0}$ because the image of $D_{-1}$ is trivial. In the $\Lambda \neq 0$ case, Torre found that $\operatorname{Ker} D_{0}$ is equivalent to the space of the Killing vectors [4] and this is also true in the $\Lambda=0$ case. The kernel of $D_{2}$ is the whole of the space $\Omega_{2}$. Hence $H^{2}$ is the subspace of $\Omega_{2}$ orthogonal to the mapping $D_{1}$, or equivalently it is the kernel of $D_{1}^{*}$. The index of the elliptic complex is defined as the alternating sum,

$$
\text { Index } \equiv h^{0}-h^{1}+h^{2}
$$

This is a topological quantity determined by the Atiyah-Singer index theorem [20]. In the $\Lambda \neq 0$ case, the elliptic complex can be reduced to a more simple form by restricting $\Omega_{1}$, the space of $\left(\delta \Sigma^{k}, \delta \omega^{k}\right)$, to the subspace $\tilde{\Omega}_{1}$ in which the first linearized equation $D \delta \omega^{k}-\frac{\Lambda}{12} \delta \Sigma^{k}=0$ in (8) is satisfied. Then $\delta \Sigma^{k}$ are linearly dependent 
on $\delta \omega^{k}$ and $\tilde{\Omega}_{1}$ becomes the space of $\delta \omega^{k}$. Besides the second linearized equation becomes trivial on $\tilde{\Omega}_{1}$ since it is derived from the first equation by operating the covariant derivative $D$ to it. Eliminating $\Sigma^{k}$ from the third linearized equation in (8) we define the operator $\tilde{D}_{1}$,

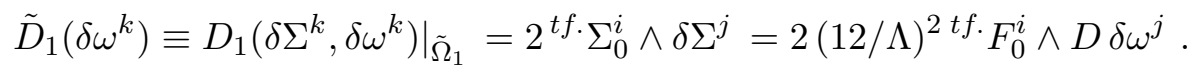

To elucidate each of spaces in (10), we introduce general spin bundles $\Omega^{m, n}$, the space of fields with spin $(m, n)$ of $\mathrm{SU}(2) \times \mathrm{SU}(2)$ 11: the space $\tilde{\Omega}_{1}$ of $s u(2)$ valued 1-forms $\delta \omega^{k}$ is $\Omega^{2,0} \otimes \Lambda^{1} \simeq \Omega^{2,0} \otimes \Omega^{1,1}$ while the space $\Omega_{0}$ of $\left(\phi^{k}, \xi^{\mu}\right)$ is equivalent to $\Omega^{2,0} \oplus \Lambda^{1} \simeq \Omega^{2,0} \oplus \Omega^{1,1}$. The elements of the image of $\tilde{D}_{1}$ are 4-forms with symmetric trace-free $\mathrm{SU}(2)$ indices, that is, with the spin $(4,0)$. Hence the space $\Omega_{2}$ becomes $\Omega^{4,0} \otimes \Lambda^{4} \simeq \Omega^{4,0}$. In terms of these spin bundles, the elliptic complex (10) is written as 2

$$
\begin{array}{cccc}
\Omega_{0} & \tilde{\Omega}_{1} & \Omega_{2} & \\
0 \stackrel{D_{-1}}{\rightarrow} & \Omega^{2,0} \oplus \Omega^{1,1} \stackrel{D_{0}}{\rightarrow} \Omega^{2,0} \otimes \Omega^{1,1} \stackrel{\tilde{D}_{1}}{\rightarrow} \Omega^{4,0} \stackrel{D_{2}}{\rightarrow} 0 .
\end{array}
$$

By applying the Atiyah-Singer index theorem to the elliptic complex, we obtain

$$
\text { Index }=\int_{M_{4}} \frac{\operatorname{ch}\left(\Omega^{2,0} \oplus \Omega^{1,1} \ominus \Omega^{2,0} \otimes \Omega^{1,1} \oplus \Omega^{4,0}\right) \operatorname{td}\left(T M_{4} \otimes \mathbf{C}\right)}{\mathrm{e}\left(T M_{4}\right)}=5 \chi-7 \tau,
$$

where ch, e and td are the Chern character, Euler class and Todd class of the various vector bundles involved. Therefore the alternating sum of $h^{i}$ in (13) is determined by the Euler number $\chi$ and Hirzebruch signature $\tau$. By changing $\tau \rightarrow|\tau|$, this index can be also adopted to manifolds with the opposite orientation. If $\Lambda>0$, as shown by Torre 画, $h^{1}$ and $h^{2}$ are found to be zero. Therefore from (13) and (16), the dimension $h^{0}$ is equal to the index,

$$
h^{0}=5 \chi-7 \tau, \quad h^{1}=h^{2}=0 .
$$

The value of $h^{0}$, the dimension of the Killing vector space, agrees that obtained by a different method in [21]. On the contrary if $\Lambda<0, h^{0}$ becomes zero [4,

\footnotetext{
${ }^{2}$ The space $\Omega_{0}$ in the complex of Torre is equivalent to $\Omega^{2,0} \oplus \Omega^{2,0} \oplus \Omega^{0,0}$, which seems slightly different from ours, $\Omega^{2,0} \oplus \Omega^{1,1}$ up to harmonic parts of the former. This leads to different results of the index.
} 
although $h^{1}$ and $h^{2}$ are not completely determined:

$$
h^{0}=0, \quad h^{2}-h^{1}=5 \chi-7 \tau .
$$

Well known examples of compact conformally self-dual Einstein manifolds with positive cosmological constant are the four-sphere $S^{4}$ with the standard metric, and the complex projective two-space $C P^{2}$ with the Fubini-Study metric. From (17), $h^{0}$ for $S^{4}$ and $C P^{2}$ are 10 and 8 respectively. These examples are important because of the following theorem of Hitchin [21].

Let $M_{4}$ be a compact conformally self-dual Einstein manifold. Then

(i) If $R>0(\Lambda>0), M_{4}$ is either isometric to $S^{4}$, or to $C P^{2}$, with their standard metrics;

(ii) If $R=0(\Lambda=0), M_{4}$ is either flat or its universal covering is the $K 3$ surface with the Calabi-Yau metric.

In the $\Lambda=0$ case, the $\mathrm{SU}(2)$ spin connection $\omega^{k}$ can be gauged away when $M_{4}$ is simply connected and then instanton equations reduce to

$$
d \Sigma^{k}=0, \quad{ }^{t f} \cdot \Sigma^{i} \wedge \Sigma^{j}=0 .
$$

These equations give the Ricci-flat condition and determine $\Sigma^{k}$ to be a trio of closed Kähler forms [5], [10]. In Ref. [22], Plebanski used these equations to derive his 'heavenly equations'. A manifold which satisfies (19) is called hyper-Kählerian. The $K 3$ surface is a unique simply connected compact manifold with such a hyperKähler structure. The elliptic complex (10) now describes the deformation of the three Kähler forms and it can be decomposed to deformations of metric and complex structures. A careful examination of the intersection part of these deformations reveals that the dimension of the moduli space of Kähler forms on the $K 3$ surface is 59 21.

In this paper, we have presented a topological version of 2-form Einstein gravity in four dimensions. For a compact manifold, we have defined the elliptic complex associated with the moduli space of our theory. By applying the Atiyah-Singer 
index theorem in the $\Lambda \neq 0$ case, we have evaluated the index of the elliptic complex.

When the dimension of the moduli space is non-zero, as occurs when $\Lambda=0$, there arise fermionic zero-modes the number of which is equal to the dimension, and these make the partition function trivial. To avoid this we need some functional $\mathcal{O}$ which absorbs the zero-modes. If one calculates the vacuum expectation value of the 'observable' $\mathcal{O}$, then it may provide non-trivial information such as a differential invariant to distinguish differential structures on conformally self-dual Einstein manifolds. Such a functional $\mathcal{O}$ is required to be BRST invariant to preserve the topological nature of the theory and may be obtained from the BRST descendant equations as in two-dimensional topological gravity [23].

It would be intriguing to study the $\Lambda=0$ case since the relation of fourdimensional (Riemann) self-dual gravity and two-dimensional conformal field theory has been investigated. In fact, Park showed that the former arises from a large $\mathrm{N}$ limit of the two-dimensional sigma model with $\mathrm{SU}(\mathrm{N})$ Wess-Zumino terms only [24]. Our topological model will be useful to understand the relation and to develop the self-dual gravity.

We are grateful to Q-Han Park and N. Sakai for useful discussions. We also acknowledge P. Crehan for reading the manuscript.

\section{References}

[1] E. Witten, Phys. Lett. B206 (1988) 601.

[2] E. Witten, Commun. Math. Phys. 117 (1988) 353.

[3] M.J. Perry and E. Teo, Cambridge preprint, DAMTP R92/42 (1992). 
[4] C.G. Torre, Phys. Rev. D41 (1990) 3620; Phys. Lett. B252 (1990) 242; J. Math. Phys. 31 (1990) 2983; A Deformation Theory of Self-Dual Einstein Spaces, preprint, (Sept., 1991).

[5] H. Kunitomo, Mod. Phys. Lett. A6 (1991) 2389.

[6] R. Myers and V. Periwal, Nucl. Phys. B361 (1991) 290.

[7] A. Nakamichi, A. Sugamoto and I. Oda, Phys. Rev. D44 (1991) 3835;

I. Oda and A. Sugamoto, Phys. Lett. B266 (1991) 280.

[8] D. Anselmi and P. Frè, Nucl. Phys. B392 (1993) 401; SISSA preprint, SISSA73-93-EP (1993).

[9] H.Y. Lee, A. Nakamichi, and T. Ueno, Phys. Rev. D47 (1993) 1563.

[10] R. Capovilla, J. Dell, T. Jacobson and L. Mason, Class. Quantum Grav. 8 (1991) 41.

[11] R. Penrose and W. Rindler, 'Spinors and Space-time' Vols. I,II (Cambridge University Press, Cambridge, 1984).

[12] L. Baulieu and I.M. Singer, Nucl. Phys. B (Proc. Suppl.) 5B (1988) 12.

[13] M. Blau and G. Thompson, Ann. of Phys. 205 (1991) 130; Phys. Lett. B255 (1991) 535.

[14] G.T. Horowitz, Commun. Math. Phys. 125 (1989) 417.

[15] G. t'Hooft, Phys. Rev. D14 (1976) 3432.

[16] J. Samuel, Class. Quantum Grav. 5 (1988) L123.

[17] E.S. Fradkin and G.A. Vilkovisky, Phys. Lett. B55 (1975) 224;

I.A. Batalin and G.A. Vilkovisky, Phys. Lett. B69 (1977) 309.

[18] M. Henneaux, Phys. Rep. 26 (1985) 1.

[19] M. Abe, A. Nakamichi and T. Ueno, in preparation.

[20] P. Shanahan, 'The Atiyah-Singer Index Theorem: An Introduction' (SpringerVerlag, Berlin, 1978).

[21] A.L. Besse, 'Einstein manifolds' (Springer-Verlag, Berlin, heidelberg, 1987). 
[22] J.F. Plebanski, J. Math. Phys. 16 (1975) 2395.

[23] E. Witten, Nucl. Phys. B340 (1990) 281.

[24] Q-Han Park, Int. J. Mod. Phys. A7 (1992) 1415. 